\title{
Direct Measure of Strain and Electronic Structure in GaAs/GaP Core-Shell Nanowires
}

\author{
Mohammad Montazeri, ${ }^{\dagger}$ Melodie Fickenscher ${ }^{\dagger}{ }^{2}$ Leigh M. Smith, ${ }^{\dagger}$ Howard E. Jackson, ${ }^{*},{ }^{\dagger}$ \\ Jan Yarrison-Rice, ${ }^{\ddagger}$ Jung Hyun Kang, ${ }^{\S}$ Qiang Gao, ${ }^{\S}$ H. Hoe Tan, ${ }^{\S}$ Chennupati Jagadish, ${ }^{\S}$ \\ Yanan Guo," Jin Zou," Mats-Erik Pistol, ${ }^{\perp}$ and Craig E. Pryor"
}

${ }^{\dagger}$ Department of Physics, University of Cincinnati, Cincinnati, Ohio 45221-0011, Department of Physics, Miami University, Oxford, Ohio 45056, ${ }^{\S}$ Department of Electronic Materials Engineering, Research School of Physics and Engineering, The Australian National University, Canberra, ACT 0200, Australia, "Materials Engineering and Centre for Microscopy and Microanalysis, The University of Queensland, Brisbane, QLD 4072, Australia, ${ }^{\perp}$ Solid State Physics and the Nanometer Structure Consortium, P.O. Box 118, SE-221 00, Lund University, Lund, Sweden, and Optical Science and Technology Center and the Department of Physics, University of Iowa, Iowa City, 54242

\begin{abstract}
Highly strained GaAs/GaP nanowires of excellent optical quality were grown with 50 nm diameter GaAs cores and 25 $\mathrm{nm}$ GaP shells. Photoluminescence from these nanowires is observed at energies dramatically shifted from the unstrained GaAs free exciton emission energy by $260 \mathrm{meV}$. Using Raman scattering, we show that it is possible to separately measure the degree of compressive and shear strain of the GaAs core and show that the Raman response of the GaP shell is consistent with tensile strain. The Raman and photoluminescence measurement are both on good agreement with 8 band k.p calculations. This result opens up new possibilities for engineering the electronic properties of the nanowires for optimal design of one-dimensional nanodevices by controlling the strain of the core and shell by varying the nanowire geometry.
\end{abstract}

KEYWORDS Strained nanowires, core-shell nanowires, photoluminescence, Raman

0 emiconductor nanowires have shown significant potential as building blocks for novel nanoscale devices. Their flexibility in creating both axial and radial heterostructures has resulted in the rapid demonstration of photosensors, light emitting diodes, single photon emitters, single electron transistors, and highly sensitive chemical and biological sensors. ${ }^{1-9}$

An important consideration in such heterostructures is that many lattice mismatched materials can be used because the large surface-to-volume ratio for one-dimensional wires allows the strain to be efficiently relaxed without the formation of dislocations or islands as happens in two dimensions. More importantly, the way the strain is distributed between the core and the shell depends on the geometry of the nanowire and, thus, provides an opportunity for engineering the energy profile of the strained nanowire core/shell heterostructures. The remarkable flexibility provided by such a situation has resulted in intense interest in expanding our understanding of these axial and radial heterostructures. For example, Glas has shown for axial heterostructures, ${ }^{10}$ and Raychaudhuri et al. have shown for radial heterostructures ${ }^{11}$ that there is a critical radius of the nanowire below which a segment or shell of any thickness can be grown without dislocations or defects. He et al. have shown that the performance of GaAs/GaP FETs can be optimized using the

* To whom correspondence should be addressed. E-mail: Howard.Jackson@uc.edu. Received for review: 10/22/2009

Published on Web: 02/04/2010 strain, ${ }^{12}$ Trammell et al. have shown that the strain can be optimized in Si/Ge nanowire FETs for enhanced device performance, ${ }^{13}$ and $\mathrm{Wu}$ et al. have suggested that strain might be used to create an optimal band offsets in $\mathrm{Si}$ nanowires for charge separation in photovoltaics. ${ }^{14}$ Finally, the rich complexity of the situation was recently described by Pistol and Pryor in two publications dealing with axial ${ }^{15}$ and radial $^{16}$ nanowire heterostructures of all types. For instance, they found that in certain circumstances for core-shell structures the band offsets could be varied between type I (spatially direct) and type II (spatially indirect), and in other circumstances one layer can be turned into a metal for a particular geometry (core radius relative to shell thickness) of the nanowire heterostructure. Such flexibility provides new opportunities for harnessing the physical properties of these strained nanowire heterostructures for particular applications or for illuminating new physics.

While there have been significant detailed studies of the structural behavior of InAs/GaAs, ${ }^{17-19}$ InAs/InP, ${ }^{8,20,21}$ and $\mathrm{GaN} / \mathrm{InN}^{22,23}$ strained axial and radial heterostructures, there have been relatively few experiments that directly related the change in the energy landscape with strain. Skold et al. showed that for GaAs/InGaP core-shell heterostructures that the GaAs core could be put under tensile or compressive strain by varying the InGaP composition. ${ }^{24} \mathrm{~A}$ number of axial and radial InAs/InP heterostructures have been demonstrated, ${ }^{8,25,26}$ but the strain effects are difficult to decouple 


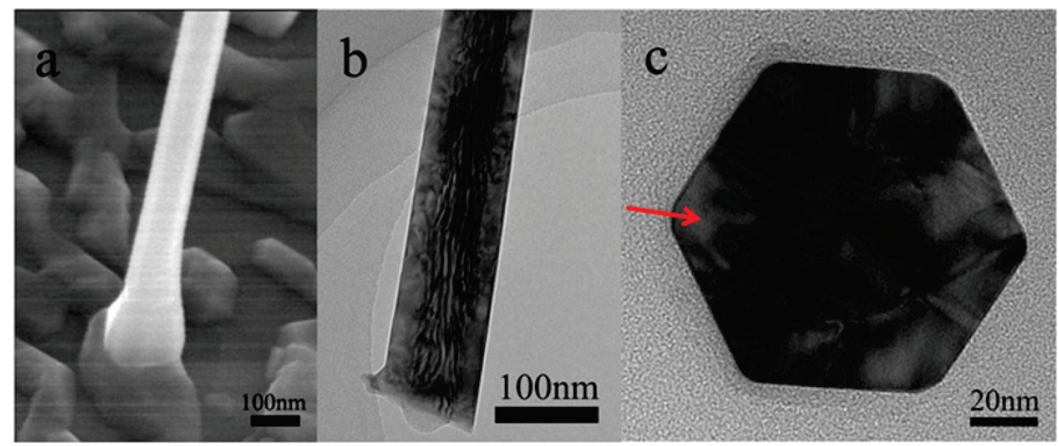

FIGURE 1. SEM micrographs of the GaAs/GaP nanowires focused on the (a) of a single nanowire. TEM bright field (BF) images taken from [112] zone axis showing the side view of the bottom section (b) of a nanowire. A typical cross-sectional TEM (XTEM) image viewing along the [111] zone axis of the bottom region (c) of a nanowire, showing a strained core-shell structure. The red arrow indicates the viewing direction of [112] for the side view image (b).

from quantum confinement effects, because there is no independent measure of the strain in each material.

In this paper, we show in heavily strained GaAs/GaP core-shell nanowires that it is possible to gain a consistent picture by directly measuring the strain in each layer using Raman scattering on a single nanowire, and correlating the change in the band structure using photoluminescence (PL) on the same single nanowire. We show that these results are consistent with what is expected from strain-dependent $\boldsymbol{k} \cdot \boldsymbol{p}$ band calculations. Most importantly, we show in a 50 $\mathrm{nm}$ GaAs core surrounded by a $25 \mathrm{~nm}$ thick GaP shell that excellent optical and structural quality of the nanowires can be obtained, even though the critical thickness for twodimensional GaP on GaAs is only 5 monolayers $(<2 \mathrm{~nm}){ }^{27}$ These results demonstrate strongly that the future of strain engineered nanowire heterostructures is very bright indeed.

Growth. The core-shell GaAs/GaP strained nanowires were grown by MOCVD on $\mathrm{Si}(111)$ ( $4^{\circ}$ miscut) substrates using gold nanocatalysts. First, a $\sim 50 \mathrm{~nm}$ thick GaAs buffer layer was grown in a two-step process: first at $400{ }^{\circ} \mathrm{C}$ with a V/III ratio of 15.4 for $60 \mathrm{~min}$, followed by a rapid $10 \mathrm{~min}$ growth at $700{ }^{\circ} \mathrm{C}$ with a V/III ratio of 154 . The buffer layer was then annealed in an arsine atmosphere at $750{ }^{\circ} \mathrm{C}$ for 15 min before removal of the growth substrate from the reactor. The Si substrate with the GaAs buffer layer was treated with poly-L-lysene (PLL) solution for $1 \mathrm{~min}$, and then a drop of $50 \mathrm{~nm}$ gold colloidal solution was applied before rinsing with deionized water. The core was grown using a two temperature growth process described by Joyce et al. ${ }^{28}$ with an initial temperature of $450{ }^{\circ} \mathrm{C}$ for $1 \mathrm{~min}$ and then the temperature was lowered to $375^{\circ} \mathrm{C}$ for a total of $30 \mathrm{~min}$. The V/III ratio was kept constantly at 46.3. Several publications have shown that the two-temperature grown GaAs nanowires result in nearly defect free cores (no twins) with little tapering and an increased carrier lifetime. ${ }^{28-30}$ The GaP shell was grown at $650^{\circ} \mathrm{C}$ for $10 \mathrm{~min}$ at a V/III ratio of 116 .

Experimental Procedure. For single nanowire studies using transmission electron microscopy (TEM) or optical spectroscopy, we used the direct transfer method to transfer the nanowire to either a holey carbon TEM grid or a marked silicon substrate for optical spectroscopy. Before the optical experiments the nanowires are mapped out on the marked silicon substrate using an optical microscope so that low temperature PL and Raman spectroscopy could be obtained on the same single nanowire.

For micro-PL measurements, the dispersed wires on the marked silicon substrate were placed in a continuous flow helium cryostat at $15 \mathrm{~K}$. The nanowires were imaged through the vacuum window using a long working length $50 \times 10.5 \mathrm{NA}$ microscope objective. A circularly polarized 532 $\mathrm{nm} \mathrm{CW}$ laser was focused to a $1.5 \mu \mathrm{m}$ spot onto the nanowire using the objective and the emitted PL was collected using the same objective. A $150 \times$ magnified image of the nanowire is imaged onto the entrance slit of a $0.25 \mathrm{~m}$ Newport imaging spectrometer and the PL dispersed using a 1200 groove/mm grating and detected by a thermoelectrically cooled CCD camera.

Room temperature Raman measurements were obtained in a similar fashion, except that the wire was imaged using a $100 \times 10.7 \mathrm{NA}$ long working length objective and the 532 $\mathrm{nm}$ laser excitation was focused to a $0.7 \mu \mathrm{m}$ spot. The scattered light was dispersed using a $600 \mathrm{~mm}$ focal length DILOR xy triple spectrometer used in subtractive mode and detected by a liquid nitrogen-cooled CCD camera.

SEM and TEM Images. The as-grown samples were characterized using field emission scanning electron microscope (FE-SEM) and TEM. The nanowires are found to be well aligned and perpendicular to the Si (111) substrate. The GaAs buffer layer forms continuous islands across the $\mathrm{Si}$ surface from which the GaAs/GaP strained nanowires form, as shown in Figure 1a. The nanowires show little tapering. Figure $1 \mathrm{~b}$ is a TEM image taken from the bottom region of a nanowire (side viewed along the [112] zone axis as indicated by the arrows in Figure 1c) and illustrates the side view of a nanowire. The GaAs core can be identified by the Moiré fringes. A comparison of the diameters of a number of GaAs cores using cross-sectional TEM (XTEM) suggests that the tapering of the GaAs/GaP nanowires is due to the tapered GaAs cores with GaP shells of uniform thickness. We note that strain contrasts can be identified at the GaAs/GaP 


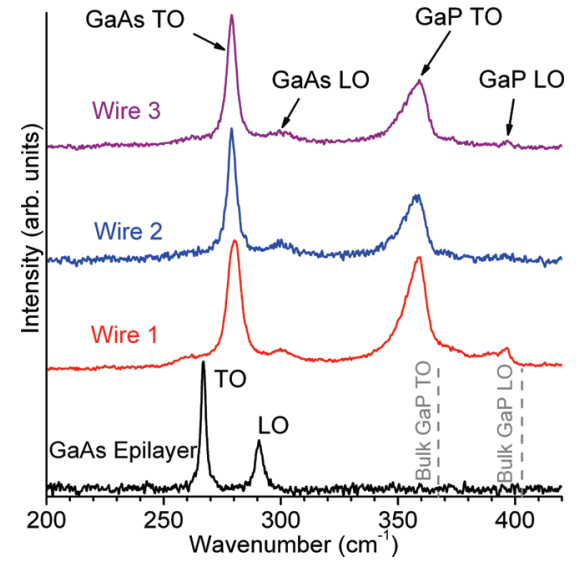

FIGURE 2. Raman spectra of GaAs/GaP nanowires (colored lines) and GaAs (black line) at room temperature. The dashed lines show TO and $\mathrm{LO}$ energies of GaP.

interfaces. Selected area electron diffraction (not shown here) confirmed that both the nanowire shell and core have the cubic zinc-blende structure.

Raman Scattering Results. Raman scattering from excitations in materials can probe a range of phenomena from magnons to plasmons to phonons. In particular, Raman scattering from phonons can provide a measurement of the stress in a material. We have carried out Raman scattering from individual nanowires at room temperature in the backscattering geometry. In a core-shell GaAs/GaP nanowire, the presence of a significant strain (both shear and hydrostatic) is expected due to the lattice mismatch of the materials $(\sim 3.6 \%){ }^{31}$ Figure 2 shows room temperature Raman scattering spectra of three examples of GaAs/GaP nanowires as well as the Raman spectrum of an unstrained GaAs epilayer grown on a GaAs substrate. The Raman spectra of the GaAs epilayer displays both a transverse optical (TO) phonon at $\sim 268 \mathrm{~cm}^{-1}$ and a longitudinal optical (LO) phonon at $\sim 291 \mathrm{~cm}^{-1}$, as expected. ${ }^{32}$

Compared to the TO and LO energies of the epitaxial GaAs, the GaAs/GaP core-shell nanowires displayed a significant shift of GaAs TO and LO phonon energies toward higher frequencies $\left(\sim 10 \mathrm{~cm}^{-1}\right)$; in addition, we observe a significant downward shift of the GaP TO and LO energies by $\sim 8$ and $\sim 6 \mathrm{~cm}^{-1}$, respectively, where the asymmetry of GaP TO response at lower energy may indicate a strain gradient in the shell. These measurements indicate that the GaAs core is under significant compressive stress and the GaP shell experiences significant tensile stress.

We used a simple model to calculate components of the strain tensor of GaAs core from Raman measurements. Because of the symmetry of the nanowire around its long axis ([111] direction), we have simplified the strain tensor to be invariant under any rotation around [111] direction. This implies that all hydrostatic components are equal and that all shear components are also equal. Thus the strain tensor in Cartesian coordinates is assumed to be

$$
\varepsilon=\left(\begin{array}{lll}
\varepsilon_{x x} & \varepsilon_{x y} & \varepsilon_{x y} \\
\varepsilon_{x y} & \varepsilon_{x x} & \varepsilon_{x y} \\
\varepsilon_{x y} & \varepsilon_{x y} & \varepsilon_{x x}
\end{array}\right)
$$

For simplicity, we have also assumed a uniform strain in the radial plane of the core without any spatial dependence, an assumption we will revisit shortly.

The secular equation for phonons can be solved for changes in the phonon energy for a zincblende crystal using phonon deformation potentials which may have different values for TO and LO modes. The degeneracy of TO modes is removed due to presence of shear strain and the scattering geometry: ${ }^{33}$

$$
\begin{gathered}
\omega_{\mathrm{T} 01}^{2}=\omega_{\mathrm{T} 00}^{2}+(p+2 q) \varepsilon_{x x}-r \varepsilon_{x y} \\
\omega_{\mathrm{T} 02}^{2}=\omega_{\mathrm{T} 00}^{2}+(p+2 q) \varepsilon_{x x}-2 r \varepsilon_{x y} \\
\omega_{\mathrm{L} 0}^{2}=\omega_{\mathrm{T} 00}^{2}+(p+2 q) \varepsilon_{x x}-r \varepsilon_{x y}
\end{gathered}
$$

where the $\omega$ 's and $\omega_{0}$ 's are the phonon energies of strained and unstrained material, $p, q$, and $r$ are the phonon deformation potentials, $\varepsilon_{x x}$ and $\varepsilon_{x y}$ are the diagonal and offdiagonal components of the strain tensor in the Cartesian coordinates system corresponding to hydrostatic and shear strain, and TO1 and TO2 are split TO modes. Note that the magnitude of TO splitting is determined only by the shear strain; thus, one may measure the shear strain by measuring the TO splitting.

Figure 3 shows a Raman spectrum of Wire 1, where Lorentzian curves are fitted to the GaAs peaks. Since we could not resolve the TO splitting, two peaks with the same

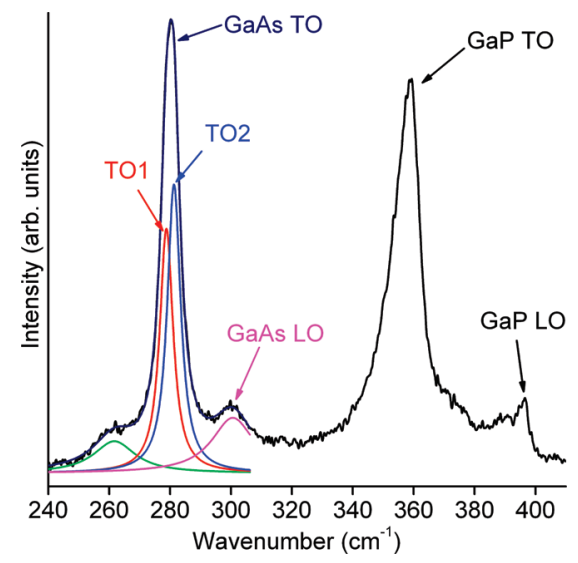

FIGURE 3. Raman response of GaAs/GaP wire 1. Lorentzian curves are fitted to GaAs response, including two split TO peaks with the same full width at half-maximum (fwhm). 


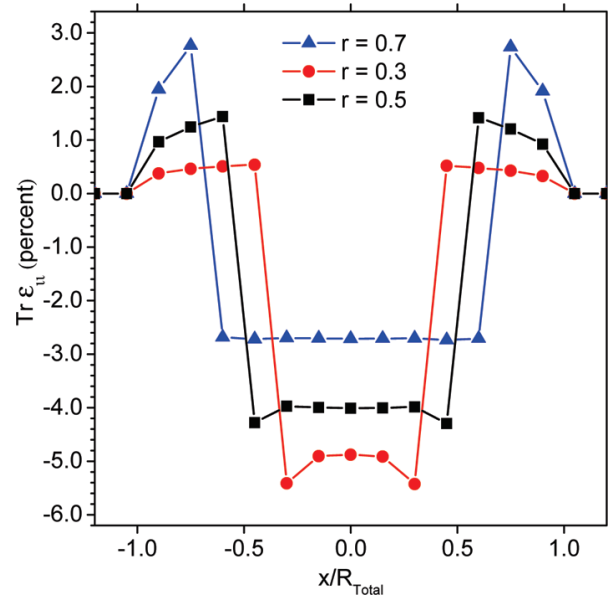

FIGURE 4. Hydrostatic strain, $\varepsilon_{x x}+\varepsilon_{y y}+\varepsilon_{z z}$, for GaAs/GaP core-shell nanowires with $R_{\text {core }} / R_{\text {total }}$ of $0.3,0.5$ and 0.7 .

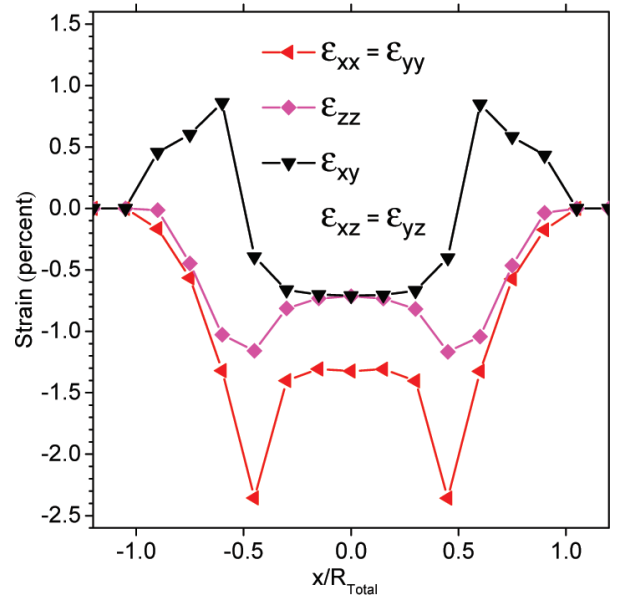

FIGURE 5. Shear strain for $R_{\text {core }} / R_{\text {total }}=0.5 \mathrm{GaAs} / \mathrm{GaP}$ nanowire.

full width at half-maximum (fwhm) are used to fit to the TO response. Determined in this way, the energies of TO1 and TO2 are 278.8 and $281.3 \mathrm{~cm}^{-1}$, respectively, which correspond to $\varepsilon_{x x}=-1.26 \%$ and $\varepsilon_{x y}=-0.69 \%$. Using eq $2 \mathrm{c}$ and the values of the strains, the energy of the LO mode is predicted to be $\omega_{\mathrm{LO}}=301.2 \mathrm{~cm}^{-1}$, which is in very good agreement with measured value of $300.6 \mathrm{~cm}^{-1}$ supporting the accuracy of the model and its assumptions. The values of phonon deformation potentials used in the calculations for GaAs were taken from ref 34. For GaP, the Raman response indicates tensile stress, as expected, but theoretical calculations show that the strain varies significantly from the outer surface to the core-shell interface and so a quantitative measure of the strain for comparison with theory is difficult.

Theoretical Modeling. Pistol and Pryor have calculated the band structure in a large variety of III-V strained core/ shell and axial nanowire heterostructures including the GaAs/ GaP core/shell structure studied here. ${ }^{15,16}$ The calculations employed continuum elastic theory to first determine the strain for the particular geometry of the nanowire. As noted in ref 16 , such strain calculations are scale invariant and so the resulting strain field only depends on the proportions of the structure and are independent of the absolute size. Once the strain fields are determined for the particular strained core/shell nanowire, an eight-band strain-dependent $\boldsymbol{k} \cdot \boldsymbol{p}$ theory was used to obtain the $\Gamma$ point energies. In Figure 4 the hydrostatic strain is shown along a line perpendicular to the nanowire axis and one of the [1 10$]$ hexagonal facets of the GaAs/GaP core-shell nanostructure. This cross section is shown for three different values of the ratio $r$, where $r$ is defined as the core radius divided by the total radius of the nanowire structure. The three cases displayed are for $r$ values of $0.3,0.5$ (corresponding to the samples in this paper), and 0.7 . The core-shell boundary can clearly be identified by the discontinuity in the strain, and the tensile strain in the shell and compressive strain in the core is as expected because of the different shell and core lattice constants.

In addition to hydrostatic strain, shear strain is present as well, as displayed in Figure 5 where the strain is in the direction [1 $1 \overline{1} 0$ ]. The different components of the shear strain are labeled and their spatial dependence shown for the case of $r=0.5$. Note that the magnitude of the shear strain is similar in magnitude to the hydrostatic strain. Thus we expect the shear strain to be important when we calculate the band structure. In Table 1, we compare the magnitude of hydrostatic and shear strain of the GaAs core determined from the Raman spectra with those from these calculations for a GaAs/GaP nanowire with $r=0.5$ for the central region of the core.

Overall, the Raman measurement for both shear and hydrostatic strain show good agreement with the above theoretical calculations. The agreement with hydrostatic strain is quite good with differences that are well within

TABLE 1. Measured LO, TO1 and TO2 Energies of GaAs Core from Room Temperature Raman Scattering and Their Corresponding Shear and Hydrostatic Strains for Three Sample GaAs/GaP Core-Shell Nanowires ${ }^{a}$

\begin{tabular}{|c|c|c|c|c|c|c|c|c|}
\hline \multirow[b]{2}{*}{ sample } & \multicolumn{6}{|c|}{ room temperature Raman measurement } & \multicolumn{2}{|c|}{ theoretical calculation $(r=0.5)$} \\
\hline & $\begin{array}{l}\text { measured TO1 } \\
\left(\mathrm{cm}^{-1}\right)\end{array}$ & $\begin{array}{c}\text { measured TO2 } \\
\left(\mathrm{cm}^{-1}\right)\end{array}$ & $\varepsilon_{x x}$ & $\varepsilon_{x y}$ & predicted LO $\left(\mathrm{cm}^{-1}\right)$ & measured $\mathrm{LO}\left(\mathrm{cm}^{-1}\right)$ & theoretical $\varepsilon_{x x}$ & theoretical $\varepsilon_{x y}$ \\
\hline wire 2 & 279.0 & 280.7 & $-1.26 \%$ & $-0.47 \%$ & 301.3 & 300.9 & $-1.34 \%$ & $-0.72 \%$ \\
\hline wire 3 & 278.1 & 279.3 & $-1.10 \%$ & $-0.35 \%$ & 300.9 & 300.3 & & \\
\hline
\end{tabular}

${ }^{a}$ The predicted LO energy from measured TO splitting is consistent with the measured LO energy. The magnitude of the strain is in good agreement with theoretical values calculated for $r=0.5$ core-shell GaAs/GaP nanowires. 


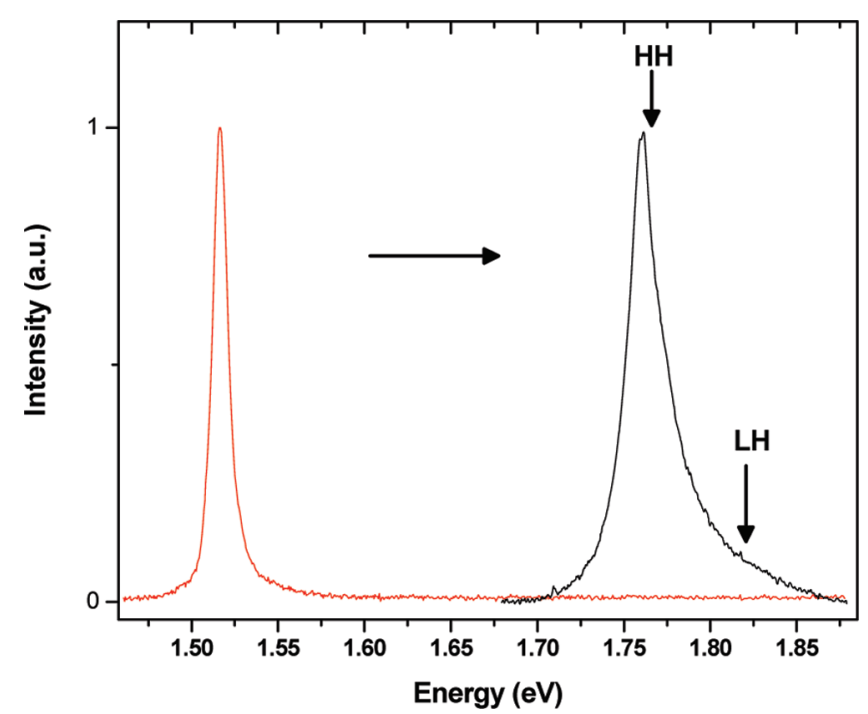

FIGURE 6. Low-temperature PL spectrum of GaAs/AlGas nanowire (red peak, left) and GaAs core of GaAs/GaP wire 3 (black curve, right). The emission line shifted $\sim 260 \mathrm{meV}$ to higher energy as a result of shell-induced strain. The vertical arrows show energies of $\mathrm{HH}$ and LH band gap, respectively, predicted from Raman measurement.

experimental error. For the shear strain there is a somewhat larger variation which may reflect sensitivity to the TO phonon fits as well as some variation in $r$. Our initial simplified analysis using approximate symmetries and phonon deformation potentials results in reasonable agreement with experiment in part because the detailed theoretical calculations (see Figures 4 and 5 above) show that within the core the hydrostatic strain components are fairly uniform and nearly equal; the same is true for the shear components.

We next consider how the presence of strain should influence the electronic structure by presenting photoluminescence measurements followed by a comparison to theoretical modeling results.

Photoluminescence: Measurements and Theory. The large hydrostatic and shear strains of the GaAs core that we have observed experimentally from Raman scattering and theoretically in modeling calculations should result in large effects on the electronic states. Furthermore, presence of the shear strain results in $\mathrm{HH}-\mathrm{LH}$ bands splitting at the $\Gamma$ point. ${ }^{35}$ Such HH-LH splitting should also occur in strained nanowires, and this has recently been seen by Lopez-Richard et al. in strained InP nanowires. ${ }^{36}$ To confirm this, microphotoluminescence measurements were performed on single nanowires that were dispersed onto a marked silicon substrate. The single nanowires were excited by $532 \mathrm{~nm}$ output from a CW laser. Care was taken to use powers for which the PL intensity grows linearly with power and also for which the line shape is minimally broadened. Nearly 20 wires were surveyed at $15 \mathrm{~K}$. Of the 20 nanowires studied, over half showed relatively strong emission at energies ranging from $1.76-1.77 \mathrm{eV}$, the remainder showed weak and broad emission possibly reflecting the presence of defects.

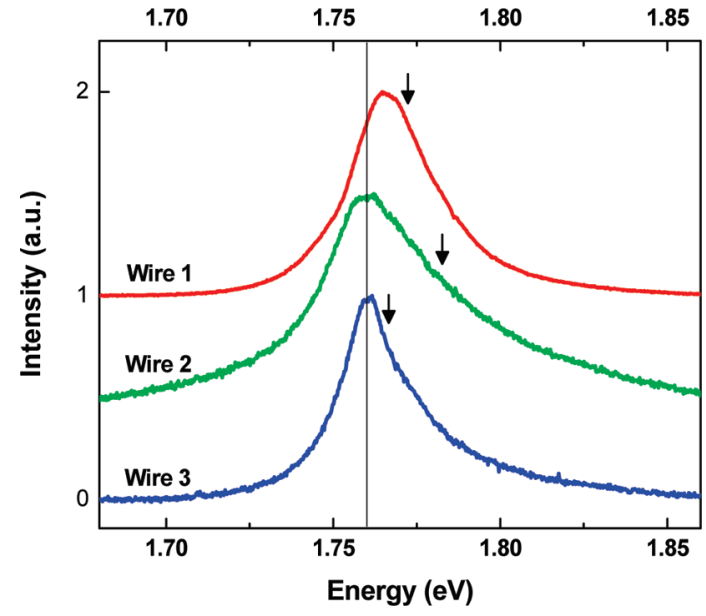

FIGURE 7. Low-temperature PL spectrum of GaAs core of GaAs/GaP wires $1-3$. The vertical arrows show energies of $\mathrm{HH}$ band gap for each wire predicted from Raman measurements of the strain. The vertical line is a guide to the eye.

Figure 6 compares the PL emission for one of these GaAs/GaP nanowires with PL emission from a high quality GaAs/AlGaAs nanowire whose core was grown under the same conditions, and whose properties were reported by Perera et al. previously. ${ }^{30}$ This PL emission from the GaAs/ GaP nanowire shows the dramatic effect of the compressive strain on the core which shifts the PL emission energy to higher energies by $\sim 260$ meV away from the expected $1.515 \mathrm{eV}$ GaAs free exciton peak which is observed in the comparison GaAs/AlGaAs nanowire. The vertical arrows show the expected emission energy for the heavy and light hole excitons one would predict given the hydrostatic and shear strains measured through the Raman scattering spectra, and using the appropriate deformation potentials to calculate the shift of the valence and conduction bands, and the splitting of the light and heavy hole bands. We do not observe PL emission from the GaP shell, which may be because recombination of the electron and hole require a phonon to take up the momentum making it significantly weaker, or due to the presence of dislocations or defects.

In Figure 7 we show photoluminescence from three different wires and show the energy position (arrow) of the heavy hole exciton based on Raman scattering results on these same wires. The agreement in the observed shift of the PL emission energy and that expected from strain measured using Raman scattering is good. We do not see any emission from the light hole band ( $\sim 150 \mathrm{meV}$ higher in energy) because at the low temperature used here no carriers thermally populate the light hole band.

We can now compare these microphotoluminescence measurements with the theoretical modeling of the band structure of the GaAs/GaP core-shell structure as calculated by the strain-dependent $\boldsymbol{k} \cdot \boldsymbol{p}$ model. Figure 8 below shows that all the bands of the GaAs cores are remarkably uniform across the nanowire except for a gradient at very 
TABLE 2. Comparison of Measured Low Temperature PL Emission Energies, Predicted Values from Raman Measurements of Strain for Three GaAs/GaP Nanowires, and Theoretical Calculations

\begin{tabular}{|c|c|c|c|c|c|}
\hline \multirow[b]{2}{*}{ sample } & \multirow{2}{*}{$\frac{\text { low temperature PL measurement }}{\text { measured emission }(\mathrm{eV})}$} & \multicolumn{2}{|c|}{ predicted from Raman measurement } & \multicolumn{2}{|c|}{ theoretical calculation $(r=0.5)$} \\
\hline & & predicted $E_{\mathrm{g}}{ }^{\mathrm{HH}}(\mathrm{eV})$ & predicted $E_{\mathrm{g}}^{\mathrm{LH}}(\mathrm{eV})$ & theoretical $E_{\mathrm{g}}{ }^{\mathrm{HH}}(\mathrm{eV})$ & theoretical $E_{\mathrm{g}}^{\mathrm{LH}}(\mathrm{eV})$ \\
\hline wire 1 & 1.766 & 1.774 & 1.893 & & \\
\hline wire 2 & 1.76 & 1.784 & 1.863 & 1.777 & 1.912 \\
\hline
\end{tabular}

close proximity of the core-shell boundary. We note, as expected, that the $\mathrm{HH}$ and $\mathrm{LH}$ are split significantly, by about $135 \mathrm{meV}$. The band gap of the GaAs core is predicted to move from its bulk value by $262 \mathrm{meV}$. The calculated band gap of the $\mathrm{HH}$ and LH bands are 1.777 and $1.912 \mathrm{eV}$, respectively.

The hydrostatic and shear strain obtained from the Raman measurements can be used with the appropriate electronic deformation potentials ${ }^{37,38}$ to predict band gap changes and the $\mathrm{HH}-\mathrm{LH}$ splitting. In Table 2 we compare these values with the $\boldsymbol{k} \cdot \boldsymbol{p}$ theoretical calculations and the PL measurement results. Since the lattice mismatch of GaAs and GaP are almost the same at room temperature and low temperature, the magnitude of both shear and hydrostatic strain are temperature independent. These results are summarized in Table 2 , which shows the peak PL emission energy for each wire, the energy position of the light and heavy hole exciton emission given the strains from Table 1, and the energy derived from the 8 band $\boldsymbol{k} \cdot \boldsymbol{p}$ calculation. In general, the agreement between the Raman and PL measurements and the theoretical calculations is excellent.

\section{CONCLUSIONS}

We have shown that it is possible to fabricate highly strained GaAs/GaP nanowires with $50 \mathrm{~nm}$ diameter GaAs cores with $25 \mathrm{~nm}$ thick GaP shells. These highly strained nanowires are of excellent optical quality and emit strongly at energies shifted dramatically by $260 \mathrm{meV}$ from the

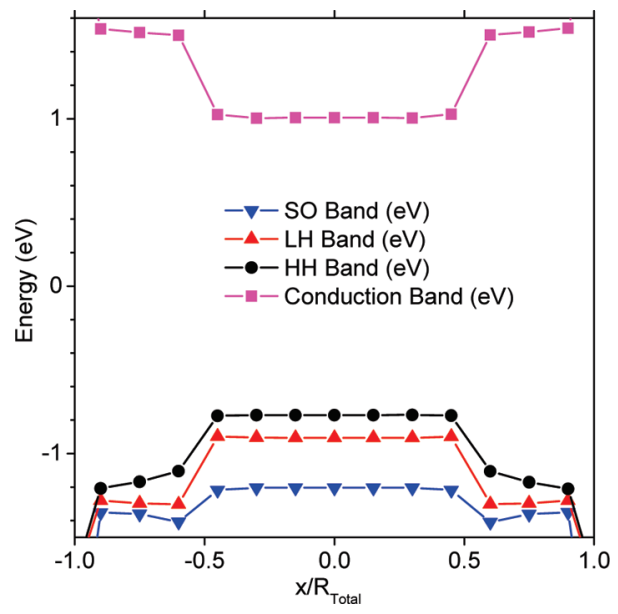

FIGURE 8. Calculated band energies of a core-shell GaAs/GaP nanowire with $r=0.5$ along [1 10$]$ direction in radial plane of nanowire. unstrained GaAs free exciton emission energy. Using Raman scattering, we show that it is possible to separately measure the degree of compressive strain as well as the shear strain in the GaAs core that splits the valence band degeneracy at the $\Gamma$ point. The Raman measurements of the GaP modes are consistent with tensile strain of the GaP shell. These results are in good agreement with 8 band $\boldsymbol{k} \cdot \boldsymbol{p}$ calculations. This result opens up new areas for investigation to utilize the ability to control the strain of the core and shell by varying the nanowire core/shell ratio in order to engineer the electronic properties of the nanowires for optimal design of one-dimensional nanodevices.

Acknowledgment. We acknowledge the financial support of the National Science Foundation through grants DMR0806700, 0806572 and ECCS-0701703, and the Australian Research Council. Australian National Fabrication Facility is acknowledged for access to the facilities used in this research. M.E.P. acknowledges the financial support of the Nanometer Structure Consortium in Lund and support by the Swedish Foundation for Strategic Research (SSF) and the Swedish Research Council (VR). C.E.P. acknowledges support from an NSF NIRT.

\section{REFERENCES AND NOTES}

(1) Maharjan, A.; Pemasiri, K.; Kumar, P.; Wade, A.; Smith, L. M.; Jackson, H. E.; Yarrison-Rice, J. M.; Kogan, A.; Paiman, S.; Gao, Q.; Tan, H. H.; Jagadish, C. Appl. Phys. Lett. 2009, 94, 193115.

(2) Minot, E. D.; Kelkensberg, F.; van Kouwen, M.; van Dam, J. A.; Kouwenhoven, L. P.; Zwiller, V.; Borgstrom, M. T.; Wunnicke, O.; Verheijen, M. A.; Bakkers, E. Nano Lett. 2007, 7, 367-371.

(3) Borgstrom, M. T.; Zwiller, V.; Muller, E.; Imamoglu, A. Nano Lett. 2005, 5, 1439-43.

(4) Patolsky, F.; Zheng, G.; Lieber, C. M. Anal. Chem. 2006, 78, 42604269.

(5) Agarwal, R.; Barrelet, C. J.; Lieber, C. M. Nano Lett. 2005, 5, 917 920 .

(6) Tian, B. Z.; Zheng, X. L.; Kempa, T. J.; Fang, Y.; Yu, N. F.; Yu, G. H.; Huang, J. L.; Lieber, C. M. Nature 2007, 449, 885-U8.

(7) Fang, Q.; Gradecak, S.; Yat, L.; Cheng-Yen, W.; Lieber, C. M. Nano Lett. 2005, 5, 2287-91.

(8) Fuhrer, A.; Froberg, L. E.; Pedersen, J. N.; Larsson, M. W.; Wacker, A.; Pistol, M. E.; Samuelson, L. Nano Lett. 2007, 7, 243-246.

(9) Wallin, D.; Fuhrer, A.; Froberg, L. E.; Samuelson, L.; Xu, H. Q.; Hofling, S.; Forchel, A. Appl. Phys. Lett. 2007, 90, 172112.

(10) Glas, F. Phys. Rev. B 2006, 74, No. 121302-1-4.

(11) Raychaudhuri, S.; Yu, E. T.J. Appl. Phys. 2006, 99, No. 114308 $1-7$.

(12) He, Y. H.; Zhao, Y. N.; Fan, C.; Kang, J. F.; Han, R. Q.; Liu, X. Y. IEEE Trans. Electron Devices 2009, 56, 1199-1203.

(13) Trammell, T. E.; Zhang, X.; Li, Y. L.; Chen, L. Q.; Dickey, E. C.J. Cryst. Growth 2008, 310, 3084-3092.

(14) Wu, Z. G.; Neaton, J. B.; Grossman, J. C. Nano Lett. 2009, 9, $2418-$ 2422 .

(15) Pistol, M. E.; Pryor, C. E. Phys. Rev. B 2009, 80, No. 035306-1-14

(16) Pistol, M. E.; Pryor, C. E. Phys. Rev. B 2008, 78, No. 115319-1-12. 
NANO

(17) Paladugu, M.; Zou, J.; Guo, Y. N.; Zhang, X.; Joyce, H. J.; Gao, Q.; Tan, H. H.; Jagadish, C.; Kim, Y. Nanoscale Research Letters 2009, $4,846-849$.

(18) Paladugu, M.; Zou, J.; Guo, Y. N.; Zhang, X.; Joyce, H. J.; Gao, Q.; Tan, H. H.; Jagadish, C.; Kim, Y. Appl. Phys. Lett. 2008, 93, No. 201908-1-3.

(19) Paladugu, M.; Zou, J.; Guo, Y. N.; Zhang, X.; Kim, Y.; Joyce, H. J.; Gao, Q.; Tan, H. H.; Jagadish, C. Appl. Phys. Lett. 2008, 93, No. 101911-1-3.

(20) Samuelson, L.; Bjork, M. T.; Deppert, K.; Larsson, M.; Ohlsson, B. J.; Panev, N.; Persson, A. I.; Skold, N.; Thelander, C.; Wallenberg, L. R. Phys. E 2004, 21, 560-7.

(21) Eymery, J.; Rieutord, F.; Favre-Nicolin, V.; Robach, O.; Niquet, Y. M.; Froberg, L.; Martensson, T.; Samuelson, L. Nano Lett. 2007, 7, 2596-2601.

(22) Kim, Y. H.; Park, H. J.; Kim, K.; Kim, C. S.; Yun, W. S.; Lee, J. W.; Kim, M. D. Appl. Phys. Lett. 2009, 95, No. 033112-1-3.

(23) Dong, Y. J.; Tian, B. Z.; Kempa, T. J.; Lieber, C. M. Nano Lett. 2009, 9, 2183-2187.

(24) Skold, N.; Karlsson, L. S.; Larsson, M. W.; Pistol, M. E.; Selfert, W.; Tragardh, J.; Samuelson, L. Nano Lett. 2005, 5, 1943-7.

(25) Larsson, M. W.; Wagner, J. B.; Wallin, M.; Hakansson, P.; Froberg, L. E.; Samuelson, L.; Wallenberg, L. R. Nanotechnology 2007, 18, No. 015504.

(26) Zanolli, Z.; Pistol, M. E.; Froberg, L. E.; Samuelson, L. J. Phys.: Condens. Matter 2007, 19, 295219.
(27) Schuler, O.; Wallart, X.; Mollot, F. J. Crystal Growth 1999, 201, 280283.

(28) Joyce, H. J.; Gao, Q.; Tan, H. H.; Jagadish, C.; Kim, Y.; Zhang, X.; Guo, Y. N.; Zou, J. Nano Lett. 2007, 7, 921-926.

(29) Zhang, G. Q.; Tateno, K.; Sanad, H.; Tawara, T.; Gotoh, H.; Nakano, H. Appl. Phys. Lett. 2009, 95, 123104.

(30) Perera, S.; Fickenscher, M. A.; Jackson, H. E.; Smith, L. M.; Yarrison-Rice, J.; Joyce, H. J.; Gao, Q.; Tan, H. H.; Jagadish, C.; Zhang, X.; Zou, J. Appl. Phys. Lett. 2008, 93, 053110-053113.

(31) Osamura, K.; Murakami, Y.Jpn. J. Appl. Phys. 1971, 10, 835.

(32) Gant, T. A.; Shen, H.; Flemish, J. R.; Fotiadis, L.; Dutta, M. Appl. Phys. Lett. 1992, 60, 1453-1455.

(33) Cerdeira, F.; Buchenauer, C. J.; Pollak, F. H.; Cardona, M. Phys. Rev. B 1972, 5, 580.

(34) Wickboldt, P.; Anastassakis, E.; Sauer, R.; Cardona, M. Phys. Rev. B 1987, 35, 1362-1368.

(35) Pollak, F. H.; Cardona, M. Phys. Rev. 1968, 172, 816.

(36) Lopez-Richard, V.; Gonzalez, J. C.; Matinaga, F. M.; Trallero-Giner, C.; Ribeiro, E.; Dias, M. R. S.; Villegas-Lelovsky, L.; Marques, G. E. Nano Lett. 2009, 9, 3129-3136.

(37) Nolte, D. D.; Walukiewicz, W.; Haller, E. E. Phys. Rev. Lett. 1987, 59, 501-504.

(38) Qiang, H.; Pollak, F. H.; Hickman, G. Solid State Commun. 1990, 76, 1087-1091.

() 2010 American Chemical Society

886

DOI: 10.1021/nl903547r | Nano Lett. 2010, 10, 880--886 